\title{
Preliminary Report on a Spiritually-Based PTSD Intervention for Military Veterans
}

Vincent R. Starnino ${ }^{1,2}$, Clyde T. Angel ${ }^{2}$, John E. Sullivan ${ }^{3,4}$, Donna L. Lazarick², Licci D. Jaimes ${ }^{5}$, John P. Cocco ${ }^{1}$, and Louanne W. Davis ${ }^{2,5}$.

1. Indiana University School of Social Work (IUPUI), 902 W. New York St., Indianapolis, IN, 46202, USA

2. Richard L. Roudebush VA Medical Center, 1481 West 10th Street, Indianapolis, IN, 46202, USA

3. Indiana University Conscience Works Project, Bloomington, IN, 46202, USA

4. Indiana University Medical School, Indianapolis, IN, 46202, USA

5. Virginia Garcia Memorial Health Center, McMinnville, OR, USA

6. Department of Psychiatry, Indiana University School of Medicine, Indianapolis, IN, 46202, USA

\begin{abstract}
Military veterans can experience spiritual/religious struggles such as weakening of beliefs, loss of meaning, increased guilt, difficulty forgiving, and moral challenges as a result of military trauma. While mainstream treatments (e.g., exposure therapy) have been shown to be effective for many, they often fail to address these issues adequately. This paper describes an 8-session spiritually-based group intervention designed to treat trauma-related spiritual wounds among military veterans. A program evaluation conducted with 24 veterans revealed significant reductions in PTSD symptoms, spiritual injury, and negative religious coping from pretest to posttest. The findings support the need for additional PTSD treatment approaches.
\end{abstract}

This is the author's manuscript of the article published in final edited form as:

Starnino, V. R., Angel, C. T., Sullivan, J. E., Lazarick, D. L., Jaimes, L. D., Cocco, J. P., \& Davis, L. W. (2019). Preliminary Report on a Spiritually-Based PTSD Intervention for Military Veterans. Community Mental Health Journal, 55(7), $1114-1119$. https://doi.org/10.1007/s10597-019-00414-8 


\section{INTRODUCTION}

Posttraumatic stress disorder (PTSD) is a critical mental health problem among military veterans and constitutes a substantial proportion of the burden of illness, affecting as many as 14 to $16 \%$, with a considerable proportion experiencing disabling symptoms for years (Gates et al. 2012). Apart from the significant psychological and financial costs to individuals with PTSD and their families, there is also a steep cost to society in terms of entitlements and lost productivity.

Exposure therapy and cognitive processing therapy are the most commonly used PTSD treatments for active military personnel and veterans. While these have been shown to be effective, dropout rates remain high for current PTSD treatments. Additionally, significant numbers remain symptomatic despite pharmacotherapy and psychotherapy (Erbes et al. 2009; Steenkamp et al. 2015). Furthermore, it may take many years for veterans to seek trauma treatment due to factors such as avoidance (a hallmark PTSD symptom), fears that directly addressing the trauma will make matters worse, and stigma related to PTSD (Erbes et al. 2009; Steenkamp et al. 2015). There is a need for additional treatment options, including those that do not emphasize detailed verbal processing of the trauma (i.e., in a Department of Veterans Affairs study that included 1931 veterans, nearly $36 \%$ of those who dropped out of exposure therapy did so due to experiencing exacerbated symptoms as a result of direct trauma processing; Eftekhari et al. 2013). It is also important to address treatment targets that are critical to recovery from PTSD, but may not receive adequate emphasis in currently available PTSD treatments (Steenkamp et al. 2015).

Spirituality and religion have been shown to be helpful resources for dealing with a variety of trauma related events including cancer, HIV, bereavement, rape, and sexual abuse (Shaw et al. 2005). There is evidence that spiritual and/or religious beliefs can be important factors in how veterans/military personnel cope following trauma (Wortmann et al. 2011). In a survey of active military, over $70 \%$ reported spirituality/religion as moderately to very important in their lives (Military Leadership Diversity Commission 2010). Although Veterans rely on spirituality as a resource to achieve acceptance, develop a support network, make meaning, and engage in self-growth 
(Pargament and Sweeney 2011), they commonly experience spiritual/religious struggles following military trauma (Fontana and Rosenheck 2004; Kopacz and Connery 2015; Litz et al. 2009). Challenges include a weakening of spiritual/religious beliefs, loss of meaning; increased guilt, difficulties with grieving and forgiving, and moral and ethical challenges. This paper describes an innovative program developed by members of our research team, called "Search for Meaning" (SFM), designed to treat trauma-related spiritual wounds. The program does not involve direct trauma processing but instead focuses on themes such as spiritual wounding and shattered spiritual beliefs, resolution of anger, forgiveness, traumatic grief, meaning making, and the rebuilding of one's spiritual foundation. We report on program evaluation findings of 24 veteran participants who completed the intervention.

\section{BRIEF SUMMARY OF THE LITERATURE}

When it comes to spiritual wounding, the metaphor of an earthquake may best describe what occurs for some veterans who experience military related trauma; it can feel like one's very foundation has been shaken. Related terms used in the literature to describe this phenomenon include "spiritual red flags" (Foy and Drescher 2015), and "moral injury" (Litz et al. 2009). Foy and Drescher (2015) used the term "spiritual red flags" to refer to various spiritually-related challenges that may be encountered after experiencing combat trauma, such as loss of faith. The authors theorized that loss of faith results when one's spiritual coping resources are overwhelmed by the magnitude of the trauma event(s). When individuals are unable to assimilate the trauma event(s) into their existing schema, or adapt their existing schema, they are susceptible to over-accommodation, which may result in setting aside spiritual beliefs altogether.

"Moral injury" represents internal conflict that can occur when one is involved in acts that compromise one's moral values (Litz et al. 2009). For many, deep-seated beliefs about what is right and wrong are related to spiritual beliefs (e.g., the world is good or fair; God is benevolent; innocent people are protected). Moral injury occurs when these deeply held assumptions are shattered by military trauma. Combat situations involving collateral damage (injury and death of innocent people or civilians, including children) are believed to create an especially high risk of internal dissonance related to moral beliefs (Litz et 
al. 2009). Resentment from being coerced into engaging in acts that betray deeply held values may grow into strong feelings of mistrust, anger, resentment, guilt, and shame (Currier et al. 2015). A related area of struggle that can have spiritual overtones includes difficulty forgiving oneself (e.g., for acts of commission or omission) and/or other people (e.g., for perceived acts of betrayal), or a higher being (e.g., anger at God for allowing the trauma event to happen; Foy and Drescher 2015).

We present two studies that establish links between spirituality and PTSD among military veterans. The first, a longitudinal study of 532 veterans engaged in a 60 day residential PTSD treatment program, examined how both adaptive and maladaptive aspects of spirituality at intake predict PTSD symptom severity at discharge (Currier et al. 2015). Findings revealed that reliance on adaptive spirituality (e.g., engaging in regular spiritual practices; looking to a higher being as a source of strength) predicted lower levels of PTSD symptoms at discharge. Conversely, spiritual struggles (e.g., feeling abandoned or punished by a higher being; experiencing a weakening of one's spiritual beliefs) predicted higher symptom severity at discharge. This study suggests that trauma treatment may be enhanced by "understanding the possible spiritual context of trauma-related concerns among veterans who possess spiritual resources or are somehow struggling in this domain" (p. 62). In the second study, Fontana and Rosenheck (2004) examined the connection between combat trauma, religious faith, and service use for 385 military veterans receiving PTSD treatment at Veterans' Administration (VA) facilities. They found that the act of killing others or believing that one failed to prevent the death of others was connected to weakening of religious faith. In turn, those with weakened religious faith were more likely to be high mental health service users at 1 year follow-up. The authors concluded that loss of religious faith is common following trauma, and can rob persons of an important mechanism for making sense of the trauma and their life in general. The authors emphasize the need for PTSD treatments to address meaning-making and loss of spiritual beliefs.

\section{TREATMENT STUDIES ADDRESSING SPIRITUAL/MORAL INJURY}

Although scholars and researchers have highlighted the importance of providing veterans with PTSD opportunities to work through and reframe spiritual/religious struggles in the 
same manner that cognitive-based therapies address negative cognitions, few clinical interventions that address spiritual/existential wounds from combat trauma have been tested and documented in the literature.

One study randomized 54 military veterans to a Building Spiritual Supports group or a waitlist (Harris et al. 2011). The intervention was spirituality-based and included prayer exercises, exploration of one's relationship with a Higher Power, and the role of forgiveness in trauma resolution. Results showed a significant reduction in PTSD Checklist scores for those who participated in the spiritual intervention. Limitations were that although all participants reported trauma exposure, 35\% did not meet the suggested cutoff score for PTSD diagnosis; and while the intervention does not exclude any spiritual/religious traditions, some theistic-related concepts (i.e., prayer) may be relevant to only a portion of the veteran population.

In another randomized study, 66 veterans with combat-related PTSD took part in a 6week mantra meditation group as an adjunct to care as usual (case management and medications; Bormann et al. 2012). Those who participated in the mantra meditation intervention scored higher on existential spiritual well-being (ESWB) and lower on PTSD symptom severity at posttest. Mediation analysis showed that the mantra meditation had an indirect effect on PTSD symptom change, with ESWB being a mediating factor that partially explained improvements in PTSD symptoms.

Description of Search for Meaning Program

SFM is an 8-week psychoeducational and processing group with a spiritual/existential/cognitive focus designed to begin the journey of examining the broader concept of spiritual injury associated with combat-related posttraumatic stress, which subsumes the more specific concept of moral injury. Each session is $90 \mathrm{~min}$. The group has been offered for the past 7 years at a VA medical center in a large Midwestern metropolitan area and is led by co-therapists: a chaplain and mental health clinician both trained in trauma treatment. Group leaders espouse a variety of traditions and all spiritual belief systems, including atheism, are accepted within the group. Veterans are encouraged to explore their spiritual wounds openly, particularly those that challenge their spiritual beliefs. 
During session one, veterans introduce themselves, and ground rules (e.g., respecting others' perspectives) are discussed and agreed upon. Individual definitions and experiences of spirituality and religion are explored in addition to the concept of spiritual wounding and its corollary symptoms and effects. Session two introduces discussion of core beliefs and how they are challenged and shaped by trauma. Veterans learn to identify the emotional symptoms of their spiritual wounds. They are encouraged to own their emotional reactions to situations and take responsibility for their actions, including their own healing. They learn to appreciate the value of being still and reflecting on their experiences and actions instead of habitually avoiding their experience of the present moment or acting impulsively. Finally, they are introduced to the concept of a mantra, encouraged to develop their own, and shown how to use it in daily life.

Sessions three and four focus on anger and the underlying, deeper feelings such as sadness, betrayal, or hopelessness that often go unrecognized and unexpressed. The experience of anger and its usefulness are normalized, while pointing out that trauma and unresolved wounds from earlier in life can result in expressions of anger that are disproportionate to the precipitating event. The facilitators teach veterans to identify the underlying causes of their anger, journal about it, begin to manage it and move toward resolution using a five step process.

During session five, participants identify the source of their power in order to explore the idea of control. Veterans are encouraged to understand that they cannot control what has happened or will happen, but rather must focus on making meaning of their lives in the present. In session six, veterans discuss grief and how it affects their daily lives. This includes the ways people commonly avoid grief and possible consequences of doing so, along with how to express grief in healthy ways while having patience with themselves.

Session seven addresses forgiveness, and differentiates forgiveness from forgetting, which many veterans feel dishonors their past or people they care about. They are helped to see that this remembering can come with painful beliefs that interfere with their wellbeing. By forgiving themselves and others for things that occurred, they are more likely to have those memories without experiencing painful emotions. Veterans are reminded that they are fully in control of when and how forgiveness occurs, and are 
coached on ways to intentionally forgive themselves and others, which may also free them from trauma-related shame.

The final session serves as a review during which participants are encouraged to reflect on the material covered in the group and bring up ideas or practices they have trouble understanding or adopting so that they can be reviewed and reinforced. Veterans are also encouraged to return to group as many times as they like to help them in their journey toward healing.

\section{PROGRAM EVALUATION FINDINGS}

\section{METHODS}

The authors conducted a program evaluation of the SFM intervention, approved by the university Institutional Review Board where the first author is employed. Participants were referred to the group as part of clinical programming (treatment as usual). Data was collected over a period of approximately 2 years from five separate cohorts. All groups took place at an urban Veterans Affairs Medical center in the Midwest. All cohorts were led by a chaplain who created the program and one of two mental health practitioners. At the time this project was conducted, facilitators had developed a detailed program guide, including participant handouts (A manualized version has since been developed).

\section{MEASURES}

The PTSD CheckList for DSM-5 (PCL-5; Weathers et al. 2013), Spiritual Injury Scale (SIS; Lawson et al. 1998), and Brief RCOPE (Pargament et al. 2011) were administered at the beginning of the first group session and at the end of the last session. The PCL-5 is a 20 -item self-report measure of the 20 DSM- 5 symptoms of PTSD. Psychometrics properties of the PCL-5 have been measured among military veteran and civilian samples-high internal consistency has been found for both (Cronbach's coefficient alpha = 0.95; Pietrzak et al. 2015; Armour et al. 2015).

The SIS is a self-report instrument that investigates the degree of difficulty a person has with eight spiritual injuries: guilt, anger/resentment, sadness/grief, lack of meaning/purpose in life, despair/hopelessness, feeling that life or God has been unfair, 
worry over religious doubt or disbelief, and fear of death. Reliability and validity are acceptable (Coefficient alpha $=0.79$; Lawson et al. 1998). The Brief RCOPE is a commonly used measure of spiritual/religious coping which consists of two 7-item subscales. Positive religious coping reflects "a secure relationship with a transcendent force, a sense of spiritual connectedness with others, and a benevolent worldview," while negative religious coping reflects "underlying spiritual tensions and struggles within oneself, with others, and with the divine" (Pargament et al. 2011, p. 51). The Brief RCOPE has shown good internal consistency across a range of empirical studies for both the positive (medium alpha 0.92) and negative (medium alpha -0.81) religious coping subscales (Pargament et al. 2011).

\section{RESULTS}

A paired-samples $t$ test was conducted to evaluate the impact of the SFM group on PTSD symptoms. Twenty-four participants who met a cut-off score of 33 on the PCL-5 were included in preliminary statistical analysis. There was a statistically significant decrease in PCL-5 scores from baseline $(M=53.96, S D 11.90)$ to post group $(M=46.54, S D 17.33), t(23)=2.54, p=0.02$ (two-tailed). The mean decrease in PCL-5 scores was $7.42(\mathrm{Cl}, 1.37$ to 13.46$)$ and the Cohen's d statistic (0.62) indicated a medium effect size. A paired samples t-test revealed a statistically significant decrease in SIS scores $(\mathrm{N}=24)$ from baseline $(M=20.08, S D 4.19)$ to post group $(M=18.42, S D 4.41), t(23)=2.30, p=0.03$ (two-tailed). The mean decrease in the SIS scores was $1.67(\mathrm{Cl}, 0.17$ to 3.16$)$ and the Cohen's d statistic $(0.40)$ indicated a small to medium effect size. In regard to the brief RCOPE, no statistically significant change was found in positive religious coping when comparing baseline $(M=18.13, S D 5.99)$ to post group scores ( $M=19.26, S D 4.65), t(22)=-1.22, p=0.23$ (two-tailed). However, there was a statistically significant decrease in negative religious coping from baseline $(M=13.59, S D 6.16)$ to post group $(M=11.14, S D 4.19), t(21)=2.07, p=0.05$ (twotailed). The mean decrease in the negative religious coping scores was $2.46(\mathrm{Cl},-0.01$ to 4.92). Cohen's d statistic (0.40) indicated a small to medium effect size.

\section{POST-PROGRAM INTERVIEW}


During a post-program interview, 16 of the 24 who completed the PCL-5 indicated which SFM group topics were useful for coping with PTSD symptoms. Over $80 \%$ found the following topics areas to be useful: "defining spirituality," "self-awareness," "identifying spiritual wounds," and "healing and hope". Discussion of "the shattered soul," "survivor's guilt," and "contentment" were identified as useful by three quarters of participants. A relatively high percentage of participants $(69 \%)$ reported benefiting from exploring topics such as "understanding anger," "learning to live in the moment," "traumatic grief," and "forgiveness". Exactly half found discussions about "finding meaning" to be useful. Discussion about "belief paradigms" was the only topic area that less than half $(37 \%)$ reported as being useful.

When participants were asked what they liked best about the program, the most common answer related to group experience (e.g., being able to talk openly with other veterans). The "program overall" was the second most common answer. A few participants mentioned liking the group facilitators. When asked to identify what they liked least about the group, the two most common answers were (1) nothing at all and (2) the shortness of the program or session. Notable recommendations include: allow group members to discuss more in depth their individual issues, talk more in depth about spirituality/religion, and provide additional resources.

\section{DISCUSSION/IMPLICATIONS}

The SFM program was developed and conducted by a chaplain and mental health clinicians (psychologists; social workers) who have extensive experience working with veterans who have PTSD. Our program evaluation of the 8-week SFM program revealed that participants made significant gains in the form of reductions in self-reported PTSD symptoms, spiritual injury and negative religious coping. Participant post interviews indicated that many of the group topics were judged as helpful by most participants and that they were satisfied overall with the SFM program in its current form. These preliminary findings are relevant considering that a number of scholars are calling for attention to be paid to the spiritual/existential/moral impact of combat trauma. In comparison to the relatively few spiritually integrated PTSD interventions that have been tested with veterans, the SFM program has a more comprehensive content focus (i.e., 
goes beyond mantra practice; Bormann et al. 2012), and the findings are based on a sample that met the cut-off for PTSD (i.e., in Harris et al. 2011 study, 35\% did not meet the cut-off).

Our goal in conducting a program evaluation of the SFM intervention was to gather information about its potential effectiveness (vs. contributing to generalized knowledge). These initial program evaluation findings are encouraging and point to a need for research to more formally evaluate the SFM program. We report here on a convenience sample of individuals who met a PCL-5 cut-off indicating likely PTSD diagnosis and were willing to complete pre and post questionnaires and a program evaluation interview. Future research should include a clinical interview to establish PTSD diagnosis, and randomize participants to the SFM program or a control group, at a minimum, waitlist. Interview feedback suggests that lengthening the program 1-2 weeks would allow for more indepth discussion of topics and for participants to apply the topics more fully to their personal experiences. Enhancement of the program guide to facilitate participants' application of concepts taught at home or after the program ends and addition of booster sessions might address participants' request for additional resources. Despite limitations, this paper makes a case for taking a deeper look at spiritual-based therapies as a potential alternative and/or adjunct to current PTSD treatment. 


\section{REFERENCES}

1. Armour, C., Tsai, J., Durham, T. A., Charak, R., Biehn, T. L., Elhai, J. D., \& Pietrzak, R. H. (2015). Dimensional structure of DSM-5 posttraumatic stress symptoms: Support for a hybrid Anhedonia and Externalizing Behaviors model. Journal of Psychiatric Research, 61, 106-113. https://doi.org/10.1016/j.jpsychires.2014.10.012

2. Bormann, J. E., Liu, L., Thorp, S. R., \& Lang, A. J. (2012). Spiritual Wellbeing Mediates PTSD Change in Veterans with Military-Related PTSD. International Journal of Behavioral Medicine, 19(4), 496-502. https://doi.org/10.1007/s12529-011-9186-1

3. Currier, J. M., Holland, J. M., \& Drescher, K. D. (2015). Spirituality Factors in the Prediction of Outcomes of PTSD Treatment for U.S. Military Veterans. Journal of Traumatic Stress, 28(1), 57-64. https://doi.org/10.1002/jts.21978

4. Eftekhari, A., Ruzek, J. I., Crowley, J. J., Rosen, C. S., Greenbaum, M. A., \& Karlin, B. E. (2013). Effectiveness of National Implementation of Prolonged Exposure Therapy in Veterans Affairs Care. JAMA Psychiatry, 70(9), 949-955. https://doi.org/10.1001/jamapsychiatry.2013.36

5. Erbes, C., Curry, K., \& Leskela, J. (2009). Treatment Presentation and Adherence of Iraq/Afghanistan Era Veterans in Outpatient Care for Posttraumatic Stress Disorder. Psychological Services, 6(3), 175-183. https://doi.org/10.1037/a0016662

6. Fontana, A., \& Rosenheck, R. (2004). Trauma, Change in Strength of Religious Faith, and Mental Health Service Use Among Veterans Treated for PTSD. The Journal of Nervous and Mental Disease, 192(9), 579-584. https://doi.org/10.1097/01.nmd.0000138224.17375.55

7. Foy, D. W., \& Drescher, K. D. (2015). Faith and honor in trauma treatment for military personnel and their families. In D. F. Walker, C. A. Courtois, \& J. D. Aten (Eds.), Spiritually 
oriented psychotherapy for trauma (pp. 233-252). Washington, DC: American Psychological Association.

8. Gates, M., Holowka, D., Vasterling, J., Keane, T., Marx, B., \& Rosen, R. (2012). Posttraumatic Stress Disorder in Veterans and Military Personnel: Epidemiology, Screening, and Case Recognition. Psychological Services, 9(4), 361-382. https://doi.org/10.1037/a0027649

9. Harris, J. I., Erbes, C. R., Engdahl, B. E., Thuras, P., Murray-Swank, N., Grace, D., Ogden, H., Olson, R. H. A., Winskowski, A. M., Bacon, R., Malec, C., Campion, K., \& Le, T. (2011). The effectiveness of a trauma focused spiritually integrated intervention for veterans exposed to trauma. Journal of Clinical Psychology, 67(4), 425-438. https://doi.org/10.1002/jclp.20777

10. Kopacz, M., \& Connery, A. (2015). The Veteran Spiritual Struggle. Spirituality in Clinical Practice, 2(1), 61-67. https://doi.org/10.1037/scp0000059

11. Lawson, R., Drebing, C. E., Berg, G. E., Jones, S., \& Penk, W. (1998). The spiritual injury scale: Validity and reliability. In Unpublished paper presented at the annual meeting of the American Psychological Association, San Francisco, CA. Retrieved September 4, 2016 from http://www. spiritualassessment.com/Manual.html.

12.Litz, B. T., Stein, N., Delaney, E., Lebowitz, L., Nash, W. P., Silva, C., \& Maguen, S. (2009). Moral injury and moral repair in war veterans: A preliminary model and intervention strategy. Clinical Psychology Review, 29(8), 695-706.

\section{https://doi.org/10.1016/j.cpr.2009.07.003}

13. Military Leadership Diversity Commission. (2010). Religious diversity in the U. S. military [Issue paper \#22]. Arlington, VA: Military Leadership Diversity Commission.

14. Pargament, K., Feuille, M., \& Burdzy, D. (2011). The Brief RCOPE: Current Psychometric Status of a Short Measure of Religious Coping. Religions, 2(1), 51-76. https://doi.org/10.3390/rel2010051 
15.Pargament, K. I., \& Sweeney, P. J. (2011). Building Spiritual Fitness in the Army: An Innovative Approach to a Vital Aspect of Human Development. American Psychologist, 66(1), 58-64. https://doi.org/10.1037/a0021657

16.Pietrzak, R. H., Tsai, J., Armour, C., Mota, N., Harpaz-Rotem, I., \& Southwick, S. M. (2015). Functional significance of a novel 7-factor model of DSM-5 PTSD symptoms: Results from the National Health and Resilience in Veterans Study. Journal of Affective Disorders, 174, 522-526. https://doi.org/10.1016/j.jad.2014.12.007

17. Shaw, A., Joseph, S., \& Linley, P. A. (2005). Religion, spirituality, and posttraumatic growth: A systematic review. Mental Health, Religion \& Culture, 8(1), 1-11. https://doi.org/10.1080/1367467032000157981

18. Steenkamp, M. M., Litz, B. T., Hoge, C. W., \& Marmar, C. R. (2015). Psychotherapy for Military-Related PTSD: A Review of Randomized Clinical Trials. JAMA, 314(5), 489-500. https://doi.org/10.1001/jama.2015.8370

19. Weathers, F. W., Litz, B. T., Keane, T. M., Palmieri, P. A., Marx, B. P., \& Schnurr, P. P. (2013). The PTSD checklist for DSM-5 (PCL-5). Scale available from the National Center for PTSD at www.ptsd.va.gov.

20. Wortmann, J., Park, C., \& Edmondson, D. (2011). Trauma and PTSD Symptoms: Does Spiritual Struggle Mediate the Link? Psychological Trauma: Theory, Research, Practice, and Policy, 3(4), 442-452. https://doi.org/10.1037/a0021413 